\title{
A Framework for Maintaining Provenance Information of Cultural Heritage 3D-models
}

\author{
Martin Doerr \\ FORTH-ICS \\ Heraklion, Crete, Greece \\ martin@ics.forth.gr \\ Maria Theodoridou \\ FORTH-ICS \\ Heraklion, Crete, Greece \\ maria@ics.forth.gr
}

\author{
loannis Chrysakis \\ FORTH-ICS \\ Heraklion, Crete, Greece \\ hrysakis@ics.forth.gr \\ Christos Georgis \\ FORTH-ICS \\ Heraklion, Crete, Greece \\ georgis@ics.forth.gr
}

\author{
Anastasia Axaridou \\ FORTH-ICS \\ Heraklion, Crete, Greece \\ axaridou@ics.forth.gr \\ Emmanuel Maravelakis \\ TEl of Crete \\ Chania, Crete, Greece \\ marvel@chania.teicrete.gr
}

\begin{abstract}
The advances in 3D digitizing technology have found significant application in the Cultural Heritage domain. The systematic large-scale production of digital cultural objects, the diversity of the processes involved and the complexity of describing historical relationships among them imposes the need for innovative knowledge management to handle all the semantic information in order to monitor, manage and document the origins and derivation of digital products. Flexibility is also very important for the enrichment and sharing of the acquired knowledge. The latter can be exploited by all members of the Cultural Heritage scientific community who can take advantage of the recorded provenance information for evaluating the 3D representation, tracking reliability of production, detecting tolerance and accuracy in acquisition and processing phases, documenting digitisation techniques, studying evidence of historic and cultural events. In this paper we present a framework that supports the documentation, archiving and dissemination of data and metadata of 3D digital cultural heritage objects, enhancing the OAIS approach by permitting the ingest of all the involved data, not only the final result, during a workflow progress.
\end{abstract}

Data provenance. Virtual heritage. 3D-models production workflow.

\section{INTRODUCTION}

The preservation of cultural heritage for the future is significant in order to make it long-term accessible for humanity. To this direction the use of information technology to capture, represent, access and maintain the data supplied by archaeologists, art historians and architects recently appeared under the name of Virtual Heritage (Koller et al. 2009). Virtual Heritage or similarly Digital Culture Heritage contains three basic activities (Krassimira et al. 2012).

The first one is the digitisation. It is the process of converting physical objects into digital form. The state-of-the art technology in digitisation includes the creation of 3D-models (Maravelakis et al. 2013). The second activity is providing access to the digital heritage. This implies the exploration of information (via browsing and querying) of Cultural Heritage $(\mathrm{CH})$ objects and the required acquisition and processing phases that were needed to produce their digital representations. In the case of 3D models access could include a 3D viewer that gives a meaningful visual representation of the model. Finally the third activity is assuring longterm preservation of digital objects and of the related information on the procedures involved in their lifecycle.

Except from the maintenance of the knowledge that resulted from these three basic activities, it is important to reclaim this knowledge in order to extract useful conclusions about the origins and the derivation of digital products. The documentation of all steps of 3D model production workflow with the combination of keeping provenance information gives to $\mathrm{CH}$ scientists a powerful tool for the preservation and dissemination of cultural heritage.

This paper presents a framework that records all steps of 3D-model production. It keeps appropriate provenance metadata for all phases enabling access, browsing and advance querying on entities, objects or events that participate in the 3Dmodel production workflow.

We studied the requirements that arise from each 3D-model production phase and the potential problems that needed to be solved in order to build 
a reliable and robust solution. We suggest an approach that enables flexible recording of the provenance information and allows knowledge population and enrichment throughout the $\mathrm{CH}$ domain.

The proposed framework consists of a Repository Infrastructure which provides directory services in conjunction to semantic repository services. It applies conceptual reference schemas to metadata, supports querying and updating of the semantic information, inferencing and reasoning. The conceptual models used are CIDOC-CRM (ISO21127:2006) (Doerr 2003, CIDOC-CRM), an ontology designed to mediate contents in the area of tangible $\mathrm{CH}$ and its extension CRMdig (Doerr \& Theodoridou 2011). The last enables the capturing of the modelling and the query requirements regarding the provenance of $3 \mathrm{D}$ digital $\mathrm{CH}$ objects. The framework also provides four tools to support ingest (Reposlt), browsing and annotating (Browslt), 3D-viewing (3DS Viewer) and resolving co-reference issues (CoReflt).

This work was initiated in the 3D-COFORM project (Doerr et al. 2010) and continued and evolved in the 3D-SYSTEK project (Doerr et al. 2014).

\section{RELATED WORK}

In the last few years several approaches and systems have been proposed for effective cultural heritage content organisation, preservation, and integration. One category of systems refers to content management systems that preserve and enable easy and open access to all types of digital content including text, images, moving images, and data sets. To this category belong DSpace (DSpace) and Fedora (Fedora). DSpace is an application for managing and providing access to digital content in an open access repository most often used for managing faculty and student output. Fedora is a modular repository platform for the management and dissemination of digital content in the form of digital objects. A different approach is presented in aDore Project (Bekaert et al. 2005) that adopts the MPEG-21 DID content representation model to provide preservation and retrieval of heterogeneous multimedia content.

However, all these systems regardless of their architecture are bound to their own data management philosophy, having limited semantic representation capabilities as they use Dublin Core schema (Dublin Core) for holding metadata, and they do not support workflows of processes and cannot exploit provenance information.

The description of workflow has been covered by OAIS (Allinson 2006) model with a minimum level of provenance that requires the storing of only final products. However, this is not the case in a 3Dmodel production workflow that is not predefined, and which cannot be described as a production pipeline, but rather as a trial and error procedure with several back and forth steps, which may be performed by different people, in distant places, and sometimes in different time-frames

In 3D-COFORM project with the applied conceptual model and the encapsulated semantics in RDF, the final products are only a part of a given process and might be the result of multiple reorganisation and re-aggregation of information. To the best of our knowledge this was the first try of real time recording of all steps of 3D-production workflow. Our work is an integrated solution proposing a conceptual and implementation approach, continuing the work done in 3D-COFORM. It includes major improvements, vital new features that resulted from complete reimplementation of core components and the creation of new tools.

\section{REQUIREMENTS}

In this section we present the requirements of maintaining provenance information on all steps of $3 \mathrm{D}$-model production. The basic requirement is a conceptual and data model that fits well in a 3Dmodelling workflow. For keeping provenance there is a need for capturing semantics (in the form of metadata) for all participating entities through the entire workflow. Thus, a dynamic Semantic Network is crucial for studying evidence of historic and cultural events, providing powerful query capabilities by extracting new conclusions taking advantage of inferencing and reasoning. Dynamicity of the Semantic Network implies the presence of update and annotation functionalities.

Also, referential integrity of information is a vital need for unique identification of data preventing duplicates and solving co-reference issues (Meghini et al. 2009). Furthermore, the reliable and scalable storage is another requirement. This includes the ability of uploading or resuming large streams of data with the garbage manipulation of temporary objects.

Finally since 3D-modelling process involves several manual and automatic procedures there is a need of intelligent recording of the workflow. This could be analysed to parallel user tasking, batch loading of procedures, handling of reproducible steps for saving storage and support of consistency due to removed raw content. 


\section{CONCEPTUAL APPROACH}

\subsection{Modelling of the workflow}

The conceptual approach of data provenance relies on the CIDOC-CRM a well-established standard for cultural heritage documentation and its extension CRMdig. The ideal adaptation and the extensibility of the standard offers validity and significant flexibility on describing $\mathrm{CH}$ entities and related activity. The key entity of the CIDOC ontology is the Event. According to CIDOC, an Event "comprises changes of states in cultural, social or physical systems, regardless of scale, brought about by a series or group of coherent physical, cultural, technological or legal phenomena. Such changes of state will affect instances of E77 Persistent Item or its subclasses".

The event-centric approach is the best way to describe the 3D-modelling workflow. The procedures that take place in such a workflow are Events that track the changes of states of the involved $\mathrm{CH}$ objects resulting in new objects and procedural states. All the classes declared in the rest of the paper follow the conventions used in the CIDOC-CRM model: E and P denote CIDOC-CRM classes and properties while $D$ and $L$ denote CRMdig classes and properties respectively.

The workflow starts with the definition of a campaign/project (E7 Activity) and consists of Acquisition and Process Events. In order to model the acquisition of geometry and other visual characteristics of the physical object we use the class D2_Digitization_Process and we record attributes such as: (i) the acquisition location (instance of E53_Place class), (ii) the related dates (values for the L31_has_starting_date-time and the L32_has_ending_date-time properties), (iii) the used equipment (D8_Digital_Device), (iv) the configuration and calibration parameters and (v) the involved persons and legal bodies (instances of E21_Person and E40_Legal_Body respectively) and other entities that participate in Acquisition. The types of Acquisition depending on the applied methodology are: the laser scanning, the structured light scanning, the stereo photography, the 2D photography, the dome based scanning, etc.

Acquisition may consist of Capture Events (D2_Digitization_Process) used to record physical object's digital representations and a Documentation (D2_Digitization_Process) event that captures the acquisition planning and setup and/or the physical object's documentation.

The acquisition phase is followed by the processing of the acquired information up to the 3D-model production. The acquired information is processed with the use of specific software and particular conditions (i.e. software parameters and settings).
To describe the processing phases, we use the class D3_Formal_Derivation which "comprises events that result in the creation of a D1 Digital Object from another one following a deterministic algorithm, such that the resulting instance of digital object shares representative properties with the original object". We record the applied software and configuration, the input Data that were used and the resulted output Data. Characteristic examples of formal derivation are: 3D-modelling, 3D-content creation, 3D-point cloud processing, coding, image processing, video processing, processing with GIS, data compression, reverse engineering etc.

The devices used for acquisition and processing belong to the D8_Digital_Device class comprising of identifiable material items such as computers, scanners, cameras, etc. that have the capability to process or produce instances of D1_Digital_Object. The latter contains all the types of bit-streams and e-files representing texts, images, audios, videos, software, etc. that are used or produced in the involved 3D-modelling procedures. A particular class is the D9 Data Object used for specific instances of the D1_Digital_Object class, those being the direct result of a digital measurement or a formal derivation.

The Annotation of objects is not strictly part of the 3D-modelling workflow. Nonetheless it is provided to assign additional information to the repository content. We use the D30 Annotation Event class to describe the creation of associations between objects or areas of objects of the repository with other objects or areas, persons, places, events. The Annotation event creates the D29_Annotation_Object entity that encapsulates the connection between the concepts related in a proposition and the activities of creation, modification and deletion.

Each of the events used in our hierarchical workflow has its own properties (links to other classes) according to the class it belongs to and also complies with the class hierarchy concepts which means that it inherits properties from its super-classes. The part-of relation (P9_consists_of) is used to link events with their sub-events and all the events involved in a specific campaign/project belong to the same hierarchy and are part-of the top level Project event (E7 Activity).

\subsection{Semantic Reasoning}

The produced data from all phases of the workflow documentation are encoded according to the Resource Description Framework (RDF) standard. RDF is provided by the W3C to support structured semantic descriptions allowed to be shared and reused across application, enterprise, and community boundaries. Starting with the explicitly 
declared events and conditions of the documented workflow and then applying steps of logical premises, we finally conclude to reasonable implicit states. Hence, the semantic repository is enriched with more meaningful data.

We present an example of reasoning for the case of data-derivatives. The output-data derived from a processing event based on input-data are called data-derivatives of the input-data. When multiple processes take place sequentially they finally create chains of data-derivatives. Then every output-data in a derivation chain is considered as a data-derivative of any input-data of the former chain processes. Thus, starting with an explicit state of a sequential chain of input-output data, we conclude to a directed graph of data-derivatives. Other examples include the transitive cases of 'part-of', 'consists-of' and 'contains' relations. Events (super-events) that 'consist-of' other events (sub-events) lead to the inference of new implicit parent-child relations inside an event hierarchy.

An innovative analysis for efficient reasoning is provided with the Fundamental Categories and Relations framework (TR-429 2012). Applying this framework we take advantage of the efficiency of the inferred direct linking: semantic resources that are interconnected via complicated relation paths can be linked directly. A representative example of this approach is the dynamically inferred 'refers-to' link between actors and the digitized object of the case study presented at section 7 . In this example, the simple Fundamental Relation 'refers-to' operates as a shortcut for the different relation paths between the E22_Man-Made_Object and the E39 Actor instances.

\section{CORE INFRASTRUCTURE}

\subsection{Repository entities}

The repository entities managed in the core system are classified into three main conceptual types:

(i) Data objects: They include the produced 3Dmodels as well as any 2D-image, text or other digital material involved in the 3D-model production process.

(ii) Area objects: One or more parts or items, representing a meaningful internal set of components of the Data object which further can be annotated and conceptually related to other resources. Specific parts of 3D-models with particular interest are expressed with this entity, in order to be classified or related to other areas, models, persons, places, etc. An Area object is specified as 'primary' Area of a Data object the first time it is defined. The same area can also be pinpointed on other instances of a Data object (e.g. its derivatives) specified then as a 'propagated' Area for the related Data object instance.

(iii) Metadata objects: Sets of RDF triples that carry the information about all semantic entities related to 3D-models or involved in the 3D-model production. They are used in an event-centric logic for: (i) The step-by-step documentation of the acquisition and process events, (ii) The description of other semantic resources such as persons, legal bodies, places, devices, terminology, typology, etc. participating in events' documentation and (iii) The creation of annotations for Data, Areas of Data or any other resource of the Semantic Network.

\subsection{Architecture and design}

The core infrastructure is based on the two tier client-server architecture. The server tier is consisted of four essential modules that cooperate to satisfy the archiving and access of the information (see Figure 1):

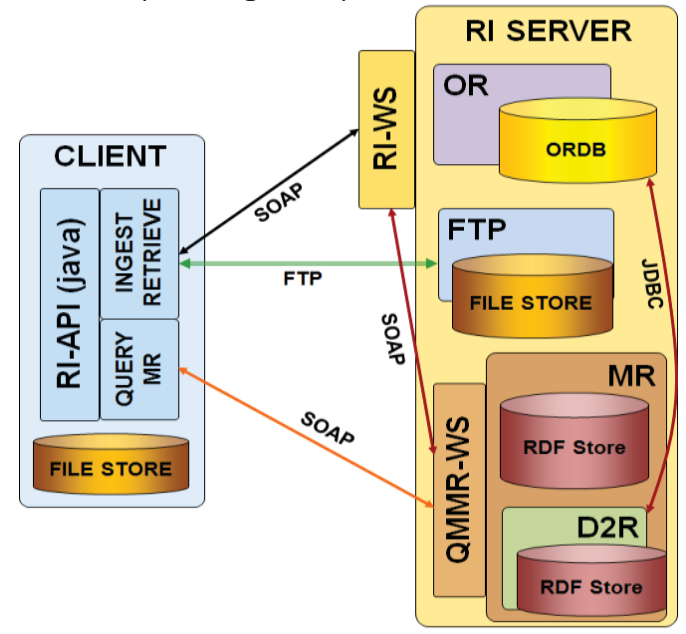

Figure 1: Architecture of the Repository Infrastructure

(i) The Repository Infrastructure Web Service (RIWS) that publishes the server's front-end interface. It is responsible for communication with the other core modules synchronizing their operation.

(ii) The Object Repository (OR) is the module that holds the registration of each object ingested to the repository. The information being recorded and stored in OR is about the file properties of the ingested objects in conjunction to the between objects relationships (i.e. Data and Metadata coupling, Metadata versions, Data with specified Areas) and other operational entities (sessions, transactions, etc).

(iii) The Metadata Repository (MR) is the module that provides a RDF triple-store to maintain the provenance knowledge. Initially, the provenance information is encoded in RDF 
and when inserted in the triple-store it gets analyzed into semantic triples of the type: subject linked via a predicate to an object. The triple constitutes the unit of knowledge. Such units are combined together to result in the building of a Semantic Network. When new triples aggregate the triple-store they constantly enrich the collected knowledge. MR internal reasoning and inferencing mechanism allows extracting the stored knowledge of the Semantic Network. Query Manager (QM) component, embedded to MR, enables the simultaneous querying of the two heterogeneous data sources, OR and MR, with a unified SPARQL interface. The returned query result is the intersection of the two distinct result sets. MR and QM are published under the QMMR Web Service: the front-end for communicating with the semantic repository infrastructure.

(iv) The FTP file server and store, serves the hosting of the uploaded files during object ingestion. The stored files are always available and can be directly accessed via FTP URLs in read-only mode. FTP provides controlled and reliable transfer of bytes, as well as the option to resume or restart an unsuccessful transferring procedure.

The client tier provides the appropriate Application Programming Interface (API) that enables the communication and interaction with the server side RIWS to accomplish archiving and querying of the desired information. This allows extensibility; the development of new applications that follow this API to take advantage of archiving and dissemination of the $\mathrm{CH}$ information. The tools provided by the framework and presented later in this paper are examples of this feature.

\subsection{System operation}

The applications that use the core system infrastructure are expected to perform the initial login step before starting interacting with the system. The created login session becomes the communication passport for every requested action from now on. To ingest a Data object to the repository, a metadata object has to be coupled with the first, carrying the related data provenance information. The ingestion of any object (Data or Metadata) regards the upload of the respective file onto the FTP file-store, the registration of ingestion in the OR catalogues and finally the semantic integration of the Metadata object into the MR.

The Metadata objects carrying the description of other resources (except from Data objects) can be ingested individually in a similar path. Update to newer versions is permitted only for Metadata objects. Versions of a Data object is a conceptually inconsistent situation since a new Data 'version' is assumed to be the result of a process regarding the provenance factor.

The Area objects when ingested in the system are uploaded onto the FTP file-store and they get registered in the OR. Additionally they are parsed to extract the specific relation (primary/propagated) with the referred Data object in order to create a semantic relation in the MR. On deleting an Area all area traces are removed from OR and MR.

Querying Data and Metadata is accomplished with the QM. Tuple queries are enabled on the entire repository or separately on either the OR or MR. Special query functions support SPARQL describe/construct and ask queries in the MR. Retrieval and download functionality is provided for all the types of the repository objects.

The content consistency is protected with the use of macroscopic (user-defined) transactions. Thus, ingestion and update actions that cause permanent changes to the repository can be undone when performed in a transaction context. Several other utilities, such as garbage collection of open inactive sessions and transactions, identifiers' uniqueness check, re-uploading options, etc. and finally the important mechanism of rebuilding the MR content from scratch by re-ingesting the entire metadata content to ensure the system stability and reliability.

\section{SUPPORTIVE TOOLS}

\subsection{Reposlt}

The Reposit tool is a standalone application that enables flexible recording of back and forth steps that comprise the 3D-model production. It reduces the possible complexity of describing a process to at most one level of sub-processes that can be assigned to different users. It supports the ingestion of acquisition and data-processing procedures with the simultaneous automatic creation of the appropriate semantic content (RDF metadata).

In addition Reposit stores all intermediate steps and potential missing files of each workflow. The tool allows as well, the update of data and metadata files and uses event templates for batch ingesting of files storing common setup for reusing in later editing.

The referential integrity of information is achieved due to application of a unified URI policy and rollback mechanisms for corrupted events or files. The tool enhances the resume capability of the core infrastructure to re-upload any incomplete files. Also, it automatically turns to update mode to 
support fixing of the last ingestion in case of unexpected failure. The only requirement to work with the tool is the RI Server on-line operation.

\subsection{Browselt}

The Browselt tool enables searching, browsing, annotating and downloading of 3D collections within the whole 3D production workflow documentation. The searching and browsing functionality is motivated by using Fundamental Relationships (TR-429 2012) between objects with the combination of free text keywords, as parameters to the final query. The resulted query is a complicated SPARQL query that can be refined any time by changing search criteria.

The navigation of query results enables a hyperlinked provenance chain of correlated entities that take part in the whole production process. Inferred results can be extracted due to reasoning capabilities of the core infrastructure. The tool also embeds annotate functionality. With the annotate functionality the user can ingest extended information about a digital object such as relation to other objects, physical condition or additional comments. The Browselt is a web-based application running in the RI Server. It has no other installation dependencies as being built on opensource technologies (HTML5 and Java Servlets).

\subsection{CoReflt}

CoReflt is a web tool enabling the users to mark identical entities in the semantic network that have different identifiers (URI duplicates: many URIs for the same resource). The repair of such duplications is subject to permissions that are granted to authorized users of the MR. The application proposes the following possible actions:

(i) Same As, that produces an annotation that correlates the duplicates as being the same resource. This solution has high complexity at query performance and we consider it as the first step for merging the URIs.

(ii) Merge, that replaces all the different URIs in the entire repository, with the one selected by the user. This function preserves all the previously used URIs as the identification history of a resource. Thus the resource instances are merged by moving their properties into one instance.

\subsection{DS Viewer}

3DS Viewer is a Web based LiDAR (Light Detection And Ranging) 3D point cloud viewer that enables the visualisation and exploitation of 3Dmodels. It is an autonomous application that runs at client-side and it is based on open-source technologies (ThreeJS: WebGL Abstraction Library
(Dirksen 2013)). It supports the most common XYZRGB format, which makes it compatible to all types of TLS (Terrestrial Laser Scanner).

3DS Viewer enables the entire point cloud to be treated as a whole object, the effective handling of large point clouds. Another significant key feature of the application is that apart from displaying color $3 \mathrm{D}$ images at various angles and different zoom levels it allows to align point clouds to $2 \mathrm{D}$ images imported in the 3D point cloud viewer from the external world.

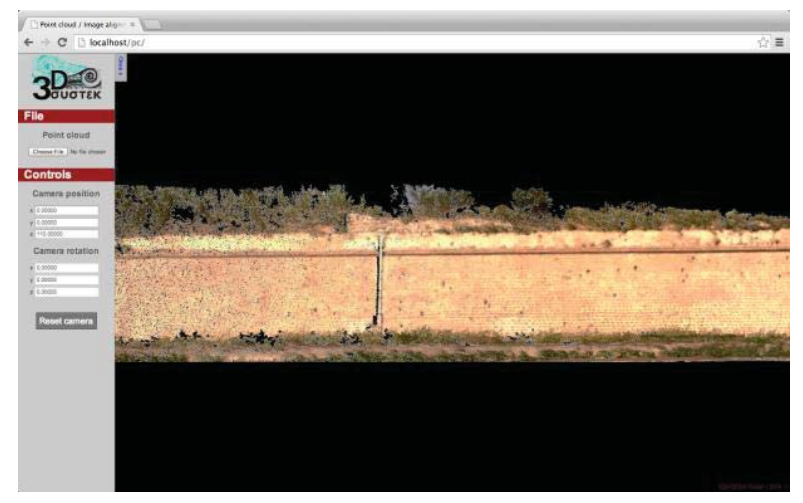

Figure 2: The user interface of 3DS Viewer

\section{CASE STUDY: THE BYZANTINE WALL OF CHANIA}

In this section we present a specific case study and how it has been supported by the proposed framework. The 28th Ephorate of Prehistoric and Classical Antiquities commissioned the Design \& Manufacturing Laboratory of the Technological Educational Institute of Crete to produce a 3D model of the Byzantine Wall of the old city of Chania in Crete. Some of the potential uses of the 3D model include (i) the advanced documentation of the monument, (ii) the creation of virtual reality presentations for touristic and educational purposes, and (iii) the study of deteriorations and damages and the support of conservation decisions. In short, the 3D model will be used to promote Cultural Heritage. The workflow of the production of the $3 \mathrm{D}$ model was the following:

(i) 3D Data Acquisition using an Optech Ilris-3D Terrestrial Laser Scanning and the Optech Controller Software. Six different scanning positions were used to cover all the examined wall area, each producing individual raw data output files ( ${ }^{*}$.blk, ${ }^{*}$.jpg and *.asc) of approx. $120 \mathrm{MB}$ size in total.

(ii) Next the Optech Parser software was used, in order to produce a point cloud of each individual scan. The raw data from the scanning process and a texture calibration parameter file was used as inputs to the software. A *.xyz point cloud file for each 
individual scan was produced, with a total file size of $280 \mathrm{MB}$.

(iii) The produced point clouds from each scan were then processed (noise reduction, obstacle removal, disconnected points removal etc), aligned and merged to create a unified point cloud (250MB) of the wall using the Polyworks IMAlign software. (iv) Finally the Polyworks IMEdit software was used for the creation and processing (smoothing, hole filling, etc) of a mesh file of the Byzantine Wall of Chania producing the final products in *.ply, *.dwg, *. stl and ${ }^{*}$.jpg formats.

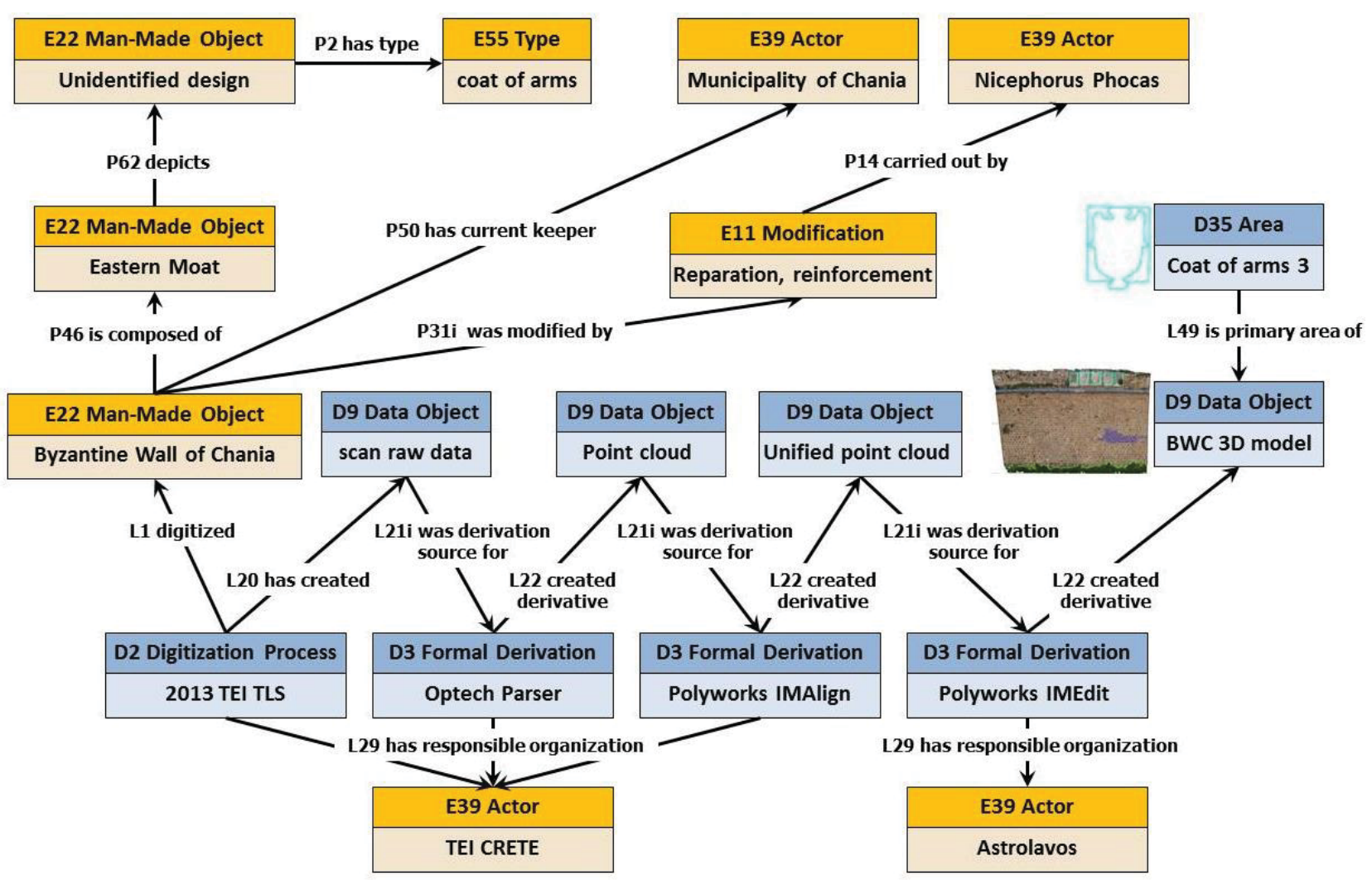

Figure 3: Simplified snapshot of the semantic network for the case study

We ingested the description of each step described above in the RI Server using the Reposit tool. A simplified snapshot of the semantic network is displayed in fig. 3 while in fig. 2 depicted the 3Dmodel in the 3DS Viewer. We also ingested an entry about the real Byzantine Wall of Chania which is composed by an Eastern Moat that depicts an unidentified design of type "coat of arms". The wall has been scanned creating scan raw data that went through three steps of processing producing the BWC 3D model. An area labeled as Coat of arms 3 has been defined on the 3D model. It is possible to avoid storing the intermediate data sets (point clouds) if they are huge. They comprise data that can be reproduced if necessary, since the system keeps detailed record of their provenance (the process method and its parameters). The metadata of deleted data continue to exist in the system, the Semantic Network has the knowledge of their production and keeps track of the derivation chain that links all the products from the acquisition to the final model. This dynamic Semantic Network can support queries that cannot be answered by traditional systems and can be efficiently formulated through the Browselt Tool.

We demonstrate representative queries to find:

Things that refer to objects of type "coat of arms": In order to answer this query, the system traverses all useful links and deduces the depiction link to all related items. The object of type "coat of arms" is depicted by: the whole Byzantine Wall of Chania, the Eastern Moat, the Unidentified design, the scan raw data, the point cloud, the unified point cloud, the BWC 3D model, and the Coat of arms 3 Area.

Actors that refer to Byzantine Wall of Chania:

Similarly, in this query the system traverses the part-of and the derivation links and collects all Actors that have created, modified, processed, curated the Wall: Municipality of Chania (the monument's current keeper), Nicephorus Phocas (responsible for repairing and reinforcing the Wall), TEI CRETE and Astrolavos (both involved in the scanning and production of the 3D model). 
Events that are the origin of the BWC 3D model of the Byzantine Wall of Chania:

This query returns the full provenance chain of the model production. The answer set includes all the events related to the production of the model: the 2013 TEI TLS, the Optech Parser, the Polyworks IMAlign and the Polyworks IMEdit final processing.

Annotations that refer to the Byzantine Wall: This query retrieves the annotation that links an area of the 3D model (Coat of arms 3) with an older image of the design comparing the two representations and actually following the deterioration of the Wall.

It becomes apparent that a seemingly simple query is internally expanded into a very complex transitive query. The system takes advantage of inference and reasoning along a huge number of semantic entities, reducing the complexity of the queries.

\section{CONCLUSIONS}

The diversity of the processes involved in largescale production of digital cultural objects and the complexity of describing the production workflow, imposes the need for innovative approach in order to monitor, manage and record the origins and derivation of digital products. The proposed framework enables intelligent documentation of 3D modelling provenance information. The reliable methodology and the implementation of appropriate infrastructure, engages the recording of the 3D modelling production with the scientific research and expression. The advancement of the produced knowledge and the dissemination throughout the $\mathrm{CH}$ domain allows a convenient reversal of time for future generations.

\section{ACKNOWLEDGEMENT}

The authors wish to thank the General Secretariat for Research and Technology of Ministry of Education and Religious Affairs, Culture and Sports in Greece for their financial support for the project: "3D-SYSTEK - Development of a novel system for 3D Documentation, Promotion, and Exploitation of Cultural Heritage Monuments via $3 D$ data acquisition, 3D modelling and metadata recording".

\section{REFERENCES}

Koller, D., Frischer, B. and Humphreys, G. (2009) Research challenges for digital archives of 3D cultural heritage models, JOCCH, Vol. 2, Issue 3, Article 7.
Krassimira, I., Dobreva, M., Stanchev, P., and Totkov, G. (2012) Access to Digital Cultural Heritage: Innovative Applications of Automated Metadata. Plovdiv University Publishing House, Plovdiv.

Maravelakis, E., Konstantaras, A. Kritsotaki, Angelakis, D., and Xinogalos, M. (2013) Analysing user needs for a unified 3D metadata recording and exploitation of cultural heritage monuments system. In ISVC 2013, Rethymno, Greece, vol. 8034, pp. 138-147.

Doerr, M. (2003) The CIDOC CRM - An Ontological Approach to Semantic Interoperability of Metadata, Al Magazine, Vol. 24, No. 3, pp.75-92

Doerr, M., and Theodoridou, M. (2011) CRMdig: A generic digital provenance model for scientific observation. TaPP'11, Heraklion, Greece.

Doerr, M., Tzompanaki, K., Theodoridou, M., Georgis, C., Axaridou, A., and Havemann, S. (2010) A repository for 3D model production and interpretation in culture and beyond. In VAST 2010, Aire-la-Ville, Switzerland, pp. 97-104.

Doerr, M., Chrysakis, I., Axaridou, A., Theodoridou, M., Georgis, C., Maravelakis, E., Konstantaras, A., and Xinogalos, M. (2014) Developing a novel system for documentation of cultural heritage monuments recorded with terrestrial 3D scanners. In CAA-GR 2014, Rethymno, Greece.

CIDOC-CRM website: http:// www.cidoc-crm.org

DSpace: http:// www.dspace.org

Fedora: http://www.fedora-commons.org

Bekaert, J., Liu, X. and Van de Sompel, H. (2005) aDORe: A modular and standards-based digital object repository at the Los Alamos National Laboratory. In JCDL '05, pp. 367-367. ACM.

Dublin Core: http://www.dublincore.org.

Allinson, J. (2006) OAIS as a Reference Model for Repositories. JISK-Report, UKOLN, UK.

Meghini, C., Doerr, M. and Spyratos, N. (2009) Managing co-reference knowledge for data integration. In Conference on Information Modelling and Knowledge Bases XX, pp. 224-244. Amsterdam, Netherlands.

TR-429 (2012) Fundamental Categories and Relationships for Intuitive querying CIDOC-CRM based repositories, ICS-FORTH, Heraklion, Greece.

Dirksen, J. (2013) Learning Three.js: The JavaScript 3D Library for WebGL. 